
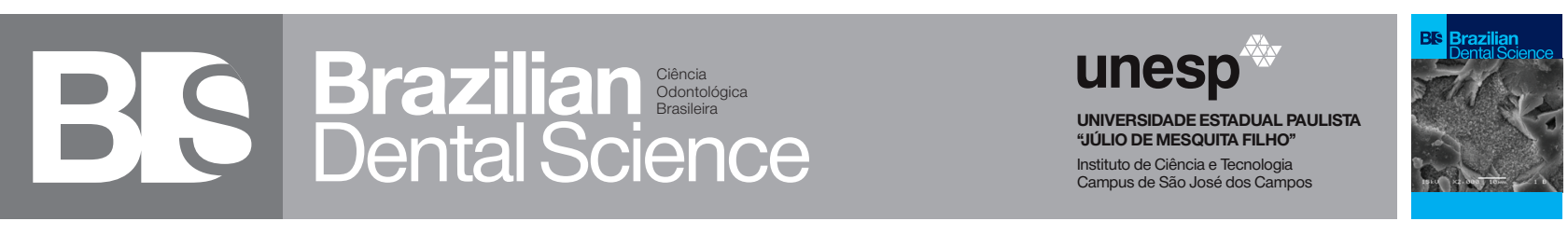

\title{
Prevalence of oral mucosal status in resident and non-resident nursing home in Isfahan city, Iran: a comparative cross-sectional study
}

Prevalência da condição da mucosa oral em idosos residente em casas de repouso ou não residente, na cidade de Isfahan, Irã: um estudo transversal comparativo

Zahra SABERI ${ }^{1}$, Fahimeh PAKRAVAN ${ }^{2}$, Leila MOHSENZADEH ${ }^{3}$

1 - Dental Research Center - Department of Oral Medicine - Dental research institiute - Isfahan University of Medical Sciences - Isfahan - Iran. 2 - Dental Implant Research Center - Department of Oral Medicine - Dental research institiute - Isfahan University of Medical Sciences Isfahan - Iran.

3 - School of Dentistry - Isfahan University Of Medical Sciences - Isfahan - Iran.

\section{ABSTRACT}

Objective: The aim of this study was to evaluate the oral mucosal status of residents nursing home and non-resident in Isfahan, Iran and compare them with each other. Material and Methods: This was a cross-sectional study comparing 281 elderly in two groups: 141 resident and 140 non-residents nursing homes in Isfahan, Iran. The selection of nursing home and all of elderly was randomly. The nonresidents elderly were randomly selected from the public places adjacent to the nursing home. Persons were examining and the relevant information recorded in a checklists. Data were analyzed by SPSS version 24 using t-test, chi square, MannWhitney, Fisher exact test. $(\alpha=0 / 05)$. Results: The sample of the case was 281 people, including 135 males (48\%) and 146 females (52\%). Of all the subjects 153 patients (54.4\%) had at least one oral lesion, 78 (55.3\%) in resident nursing home and 75 (53.6\%) in non-resident. There was no significant difference between two groups based on Chi-Square test $(\mathrm{P}>0 / 05)$. In this study, 25 different types of lesions were recorded, the most common lesion in all participants was epulis fissuratum (12.1\%). Conclusion: The results showed that the prevalence of mucosal lesions is high in the elderly living in the centers and in the non-resident. Therefore, maintaining dentures is necessary to prevent and control lesions.

\section{KEYWORDS}

Lesion; Mouth mucosa; Elderly; Nursing home; Cross-sectional study.

\section{RESUMO}

Objetivos: O objetivo deste estudo foi avaliar a condição da mucosa oral de residentes em casas de repouso e não residentes em Isfahan, Irã, e compará-los entre si. Material e Métodos: Estudo transversal que comparou 281 idosos em dois grupos: 141 casas de repouso residentes e 140 não residentes em em casas de repouso na cidade de Isfahan, Irã. A seleção da casa de repouso de idosos e todos os idosos envolvidos foi aleatória. Os idosos não residentes foram selecionados aleatoriamente nos locais públicos adjacentes ao lar de idosos. As pessoas foram examinadas e as informações relevantes registradas. Os dados foram analisados pelo SPSS versão 24 , utilizando o teste t, Qui-quadrado, Mann-Whitney, teste exato de Fisher $(\alpha=0 / 05)$. Resultados: A amostra do estudo constou de 281 pessoas, incluindo 135 homens (48\%) e 146 mulheres (52\%). Do total geral, 153 pacientes $(54,4 \%)$ apresentaram pelo menos uma lesão oral, sendo $78(55,3 \%)$ em idosos residentes na casa de repouso e 75 $(53,6 \%)$ em idosos não-residentes. Não houve diferença significativa entre os dois grupos com base no teste do Quiquadrado ( $\mathrm{p}>0.05$ ). Neste estudo, foram registrados 25 tipos diferentes de lesões, sendo que a lesão mais comum em todos os participantes foi a epulis fissuratum $(12,1 \%)$. Conclusão: Os resultados mostraram que a prevalência de lesões nas mucosas é alta nos idosos residentes nas casas de repouso e também em não residentes. Portanto, a manutenção da prótese é necessária para prevenir e controlar as lesões.

\section{PALAVRAS-CHAVE}

Lesão; Mucosa bucal; Idosos; Lar de idosos; Estudo transversal. 


\section{INTRODUCTION}

0 ral mucosal lesions include a very wide range of benign, pre-cancerous and malignant lesions. Oral health plays an important role in people's quality of life. Oral lesions can lead to discomfort, pain, interference with the functions of chewing, swallowing and speaking. In addition, they can cause to bad smell and dry mouth, which may interfere with the daily activities of patients [1]. The prevalence of oral mucosal lesion in general population globally varies significantly across different countries and areas, ranging from $4.9 \%$ to $64.7 \%$ [1,2]. According to the World Health Organization (WHO) statement in 1984, the elderly population is over 60 years of age. Due to an increase in the average age of humans, an important part of the population is made up of elderly, and need to receive more dental care [3].

Many lesions and oral conditions are associated with age, and can have significant effects on quality of life [4]. As periodontal diseases and dental problems, oral mucosal diseases are also seen in the elderly. Endodontics and followed by use of removable prosthesis, can cause some mucosal lesions such as prosthetic osteoarthritis, lumbar puncture, epulis fissuratum, and traumatic ulcer. Also, some vascular lesions (varicose veins), pre-cancerous lesions (such as leukoplakia and erythropoelacia) are more common in the elderly than non-elderly people [2]. In large cities of Iran, elderly people are maintained at the elderly care centers, and it seems that oral lesions are more prevalent due to the use of dentures, smoking, and systemic diseases [5].

Several studies have shown that elderly people living with their families are healthier due to the social and emotional support of family members and useful social relationships [6,7]. In contrast, living in elderly care center has benefits such as contact with same age people and access to nursing, health and medical facilities. On the other hand, because lack of specialized services to address all needs of elderly, these centers are not considered as comfortable living centers for the elderly [6]. Several studies have also shown that the nutritional status of residents nursing home care center is lower [6]. Malnutrition in elderly has a significant impact on their health and quality of life, including dental, oral and language health [8].

One of the first steps to plan for prevention and treatment of diseases is to determine the prevalence of various diseases in different societies. The incidence of oral lesions can be different in people resident nursing home and non-resident care centers [6]. Therefore, the aim of present study was to evaluation prevalence of oral pathological lesion in resident nursing home and non-resident in Isfahan-Iran.

\section{MATERIAL AND METHODS}

\section{Study subjects}

The study was approved in the medical ethics committee of Isfahan University of Medical Sciences, Isfahan, Iran. This cross-sectional study conducted on elderly people in Isfahan city, Iran in 1996. Inclusion criteria were people over 60 years old and have ability to perform the minimum daily activities and experience at least one year of residence nursing home. Exclusion criteria were elderly people who were not willing to cooperate, elderly people with Alzheimer's or other mental disease, as well as elderly people under special care. The non-residents were randomly selected from nearby areas of the nursing home with invitation. Therefore, 300 elderly people were selected randomly. However, several numbers of elderlies were excluded due to inaccurate and incomplete information in the questionnaires data. Finally, 281 elderly people, including 141 resident and 140 non-resident nursing home, were examined.

\section{Evaluation of oral lesions}

Data collection was performed through examination, interview and completion of the questionnaire. The collected data include demographic information (age, sex, duration of resident nursing home), health history (consumption of tobacco and alcohol, systemic diseases and drugs), oral hygiene, denture use (type and duration of use) and existing oral lesions type. In this study, natural structure diversity such 
as plicata tongue, sublingual varices, granular friedeys, lecudom, and thoros were not considered. The soft tissue lesions such as vesiculobulose lesions, exophytic lesions, white and red lesions, and pigmentation were investigated.

\section{Statistical analysis}

The data analysis was performed using SPSS version 24 (IBM Corporation, New York, USA) software. The T-test, Chi square, Mann-Whitney and Fisher's exact test were used to analyze the obtained data. P-values less than 0.05 were taken to be statistically significant.

\section{RESULTS}

The studied subjects were 281 elderly include 135 men (48\%) and 146 women (52\%), aged 60 to 95 years old. The mean age of the subjects was $69.73 \pm 8.70$ years. The mean age in resident nursing home was $71.9 \pm 5.70$ and in non-resident was $67.91 \pm 7.15$ years.

The obtained results showed that there was no significant difference in age and sex between two studied groups. The Chi square test showed that occupation and marital status were significantly different between two groups $(\mathrm{P}<0.001)$. The Mann Whitney test also showed that two groups did not have any significant difference in terms of economic and education level $(\mathrm{P}>0.429$ vs $\mathrm{P}>0.474$ ). The distribution of lesions in terms of demographic characteristics is shown in Table 1.

The most common place of mouth lesions nursing home was related to tongue. In general, 234 (84.2\%) subjects include $118(85.5 \%)$ resident nursing home and 116 (82.9\%) non-resident were diagnosed with at least one systemic disease. According to chi square test, there was no significant difference between two groups (Table 2).

Also, 249 (89.6\%) subjects include 127 (92.0\%) resident nursing home and 122 (87.1\%) non-resident were used at least one type of medicine. According to chi square test, there was no significant difference between two groups ( $\mathrm{p}>0.183$ ).

153 (54.4\%) subjects include 78 (55.3\%) resident nursing home and 75 (53.6\%) nonresident were diagnosed with at least one oral lesion. According to chi square test, there was no significant difference between two groups ( $\mathrm{p}>0.769)$.

In this study, 25 different types of lesions were observed which most common lesions were epulis fissuratum (12.1\%), dancharostomyitis (11.7\%), candidiasis (11.1\%). The most abundant lesions were related to white and red lesions (Table 3).

In average 1.01 lesions were observed in studied elderly, which was 1.09 lesions in resident nursing home and 1.07 lesions in nonresident. According to Mann-Whitney test, there was no significant difference between two groups ( $\mathrm{p}>0.639)$.

In the present study, 146 (52.1\%) elderly used denture. At least one lesion was observed in $93(63.7 \%)$ elderly with denture versus in 60 (44.8\%) elderly without denture. The Chi-Square test showed a significant difference in prevalence of lesions between elderly with denture and elderly without denture $(\mathrm{P}=0.001)$ (Table 4).

The Chi square test showed that smoking is significantly higher in residents nursing home $(29.2 \%)$ than non-resident $(15.2 \%) \quad(P=0.022)$. Also, there is a significant relationship between smoking and prevalence of lesions $(\mathrm{P}=0.014)$. The relationship between smoking and lesions such as melanosis induced by cigarette and hairy tongue was statistically significant $(\mathrm{P}=0.001)$.

The Chi square test showed that there was no significant difference in prevalence of lesions between men (57.8\%) and women (51.4\%) $(\mathrm{P}=0.281)$. However, the prevalence of hairy tongue $(\mathrm{P}=0.044)$ and melanosis induced cigarette $(\mathrm{P}=0.001)$ in men, and tattooed amalgam $(\mathrm{P}=0.025)$ in women were significant.

The t-test showed that the mean age in elderly with lesion (70.73 \pm 9.48 years) was significantly higher than elderly without lesion (68.5 \pm 8.70 years) $(\mathrm{P}=0.035)$. Also, we showed that prevalence of lesions was not related to drug use $(\mathrm{P}=0.446)$, systematic disease $(\mathrm{P}=0.358)$ and duration of resident nursing home $(\mathrm{P}=0.312)$.

The Mann Whitney's test showed that hygiene is significantly lower in resident nursing home than in non-resident $(\mathrm{P}=0.020)$. 
Table 1 - The demographic characteristics of studied elderly and prevalence of oral lesions

\begin{tabular}{|c|c|c|c|c|c|c|}
\hline \multicolumn{2}{|c|}{ Demographic characteristics } & \multirow{2}{*}{$\begin{array}{l}\text { Resident } \\
75(2 \% / 53) \\
66(8 \% / 46)\end{array}$} & \multirow{2}{*}{$\begin{array}{c}\text { Non-resident } \\
71(7 \% / 50) \\
69(3 \% / 49)\end{array}$} & \multirow{2}{*}{$\begin{array}{c}\text { Total } \\
146(0 \% / 52) \\
135(0 \% / 48)\end{array}$} & \multirow{2}{*}{$\begin{array}{c}\text { Presence of } \\
\text { lesions } \\
76(1 \% / / 52) \\
82(7 \% / 60)\end{array}$} & \multirow{2}{*}{$\begin{array}{c}\text { p-value } \\
0.143 a\end{array}$} \\
\hline Sexuality & woman man & & & & & \\
\hline Age & $\begin{array}{l}\text { 60-70 years } \\
70-80 \text { years } \\
\text { Over } 80 \text { years }\end{array}$ & $\begin{array}{l}80(0 \% / 58) \\
35(4 \% / 25) \\
23(7 \% / 16)\end{array}$ & $\begin{array}{c}96(1 \% / 70) \\
28(4 \% / 20) \\
13(5 \% / 9)\end{array}$ & $\begin{array}{c}176(0 \% / 64) \\
63(9 \% / 22) \\
36(1 \% / / 13)\end{array}$ & $\begin{array}{l}114(8 \% / 64) \\
42(7 \% / 66) \\
31(1 \% / 86)\end{array}$ & $0.066 \mathrm{~b}$ \\
\hline Marital status & $\begin{array}{l}\text { Single } \\
\text { Married } \\
\text { Wife died } \\
\text { Others }\end{array}$ & $\begin{array}{c}42(8 \% / 29) \\
11(8 \% / 7) \\
66(8 \% / 46) \\
22(15.6 \%)\end{array}$ & $\begin{array}{c}7(3 \% / / 14) \\
107(5 \% / 77) \\
21(2 \% / / 15) \\
3(2 \% / 2)\end{array}$ & $\begin{array}{c}49(6 \% / 17) \\
118(3 \% / 42) \\
87(2 \% / 31) \\
25(0 \% / 9)\end{array}$ & $\begin{array}{l}27(1 \% / / 55) \\
65(1 \% / 55) \\
49(3 \% / 56) \\
17(0 \% / 68)\end{array}$ & $0.687 a$ \\
\hline Educational status & $\begin{array}{l}\text { Illiterate } \\
\text { Elementary } \\
\text { Guidance } \\
\text { Diploma } \\
\text { Higher education }\end{array}$ & $\begin{array}{c}40(9 \% / 29) \\
38(4 \% / 28) \\
14(4 \% / 10) \\
34(4 \% / 25) \\
7(2 \% / 5)\end{array}$ & $\begin{array}{c}34(9 \% / 45) \\
50(7 \% / 35) \\
12(6 \% / 8) \\
25(9 \% / 17) \\
19(6 \% / 13)\end{array}$ & $\begin{array}{l}74(0 \% / 27) \\
88(1 \% / 32) \\
26(5 \% / 9) \\
59(5 \% / 21) \\
26(5 \% / 9)\end{array}$ & $\begin{array}{l}46(2 \% / 62) \\
48(5 \% / / 54) \\
10(5 \% / 38) \\
32(2 \% / / 54) \\
17(4 \% / 65)\end{array}$ & $0.205 b$ \\
\hline Job status & $\begin{array}{l}\text { Unemployed } \\
\text { Housewife } \\
\text { Retired } \\
\text { Employee } \\
\text { Worker } \\
\text { Free job } \\
\text { Other }\end{array}$ & $\begin{array}{c}69(4 \% / 50) \\
4(9 \% / 2) \\
45(5 \% / 13) \\
0(0 \% / 0) \\
0(0 \% / / 0) \\
0(0 \% / 0) \\
19(8 \% / 13)\end{array}$ & $\begin{array}{c}10(1 \% / 7) \\
53(9 \% / 37) \\
49(0 \% / 35) \\
10(1 \% / 7) \\
4(9 \% / 2) \\
13(3 \% / 9) \\
1(7 \% / 0)\end{array}$ & $\begin{array}{c}79(5 \% / 28) \\
57(6 \% / 20) \\
94(9 \% / 33) \\
10(6 \% / 3) \\
4(4 \% / 1) \\
13(7 \% / 4) \\
20(2 \% / 7)\end{array}$ & $\begin{array}{c}41(9 \% / / 51) \\
30(6 \% / / 52) \\
52(3 \% / / 55) \\
7(0 \% / 70) \\
3(0 \% / 75) \\
7(8 \% / 53) \\
18(9 \% / 88)\end{array}$ & $0.127 a$ \\
\hline Economic status & $\begin{array}{l}\text { Good } \\
\text { Medium } \\
\text { Weak }\end{array}$ & $\begin{array}{l}25(0 \% / 18) \\
53(1 \% / 38) \\
61(9 \% / 43)\end{array}$ & $\begin{array}{l}20(4 \% / 14) \\
70(4 \% / 50) \\
49(3 \% / 35)\end{array}$ & $\begin{array}{c}45(2 \% / / 16) \\
123(2 \% / 44) \\
110(6 \% / / 39)\end{array}$ & $\begin{array}{l}27(0 \% / 60) \\
68(3 \% / / 55) \\
63(3 \% / 57)\end{array}$ & $0.872 b$ \\
\hline
\end{tabular}

a: chi square test; b: mann-whitney test

Table 2 - The demographic characteristics of studied elderly and prevalence of oral lesions

\begin{tabular}{|c|c|c|c|c|}
\hline Systemic diseases & Resident & Non-resident & Total & p-value \\
\hline Any systemic disease & $118(5 \% / 85)$ & $116(9 \% / 82)$ & $234(2 \% / 84)$ & $\mathrm{a} 545 / 0$ \\
\hline Cardiovascular disease & $28(1 \% / 20)$ & $37(4 \% / 26)$ & $65(25 \% / 23)$ & a214/0 \\
\hline Musculoskeletal disorders & $13(4 \% / 9)$ & $12(6 \% / 8)$ & $25(0 \% / 9)$ & $\mathrm{a} 819 / 0$ \\
\hline kidney Diseases & $8(8 \% / 5)$ & $9(4 \% / 6)$ & $17(1 \% / 6)$ & a814/0 \\
\hline Infection & $0(0 \% / 0)$ & $1(7 \% / 0)$ & $1(35 \% / 0)$ & $\mathrm{b} 000 / 1$ \\
\hline Sight and hearing problems & $25(7 \% / 17)$ & $18(9 \% / 12)$ & $43(3 \% / 15)$ & a257/0 \\
\hline Endocrine diseases & $4(9 \% / 2)$ & $7(0 \% / 5)$ & $11(95 \% / 3)$ & $\mathrm{a} 362 / 0$ \\
\hline Respiratory diseases & $7(0 \% / 5)$ & $9(4 \% / 6)$ & $16(8 \% / 5)$ & $\mathrm{a} 617 / 0$ \\
\hline Blood diseases & $0(0 \% / 0)$ & $3(1 \% / 2)$ & $3(05 \% / 1)$ & b247/0 \\
\hline Cancer & $2(4 \% / 1)$ & $3(1 \% / 2)$ & $5(75 \% / 1)$ & $\mathrm{b} 000 / 1$ \\
\hline Blood pressure & $51(7 \% / 36)$ & $55(0 \% / 40)$ & $106(35 \% / 38)$ & $\mathrm{a} 570 / 0$ \\
\hline Immunological disease & $3(2 \% / 2)$ & $2(4 \% / 1)$ & $5(8 \% / 1)$ & $\mathrm{b} 684 / 0$ \\
\hline Diabetes & $18(9 \% / 12)$ & $35(0 \% / 25)$ & $53(95 \% / 18)$ & $\mathrm{a} 010 / 0^{*}$ \\
\hline Mental illness and dementia & $39(0 \% / 38)$ & $11(8 \% / 7)$ & $50(95 \% / 17)$ & $\mathrm{a} 000 / 0^{*}$ \\
\hline Alzheimer & $22(80 \% / 15)$ & $4(9 \% / 2)$ & $26(30 \% / 9)$ & $\mathrm{a} 000 / 0^{*}$ \\
\hline Digestive disease & $9(5 \% / 6)$ & $16(4 \% / 11)$ & $25(95 \% / 8)$ & a147/0 \\
\hline Allergy & $0(0 \% / 0)$ & $2(4 \% / 1)$ & $2(7 \% / 0)$ & $\mathrm{b} 498 / 0$ \\
\hline Blood fat & $8(8 \% / 5)$ & $26(6 \% / 18)$ & $34(2 \% / 12)$ & $\mathrm{a} 001 / 0^{*}$ \\
\hline Rheumatoid Arthritis & $5(6 \% / 3)$ & $7(0 \% / 5)$ & $12(2 \% / 4)$ & $\mathrm{a} 564 / 0$ \\
\hline Epilepsy & $6(3 \% / 4)$ & $2(4 \% / 1)$ & $8(85 \% / 2)$ & b173/0 \\
\hline Addiction & $2(4 \% / 1)$ & $0(0 \% / 0)$ & $2(7 \% / 0)$ & b247/0 \\
\hline Other & $6(3 \% / 4)$ & $1(7 \% / 0)$ & $7(5 \% / 2)$ & $\mathrm{b} 066 / 0$ \\
\hline
\end{tabular}

a: chi square test; b: Fisher exact test 
Table 3 - The prevalence of oral lesions in the studied elderly

\begin{tabular}{|c|c|c|c|c|}
\hline Lesions type & Resident & Non-resident & Total & p-value \\
\hline $\begin{array}{l}\text { Vesiculobullous lesions } \\
\text { 1. Herpes } \\
\text { 2. Traumatic wound } \\
\text { 3. Aphthous }\end{array}$ & $\begin{array}{l}10(6 \% / \vee) \\
1(7 \% / 0) \\
8(7 \% / 5) \\
1(7 \% / 0)\end{array}$ & $\begin{array}{l}15(7 \% / 10) \\
2(4 \% / 1) \\
11(9 \% / / v) \\
3(1 \% / 2)\end{array}$ & $\begin{array}{c}25(4 \% / 9) \\
3(0 \% / / 1) \\
19(8 \% / 6) \\
4(4 \% / 1)\end{array}$ & $\begin{array}{l}286 / 0 \mathrm{a} \\
622 / 0 \mathrm{~b} \\
466 / 0 \mathrm{a} \\
371 / 0 \mathrm{~b}\end{array}$ \\
\hline $\begin{array}{c}\text { Exophytic lesions (tumor-like) } \\
\text { 1. Epulis fissuratum } \\
\text { 2. Fibroma } \\
\text { 3. Mucocell }\end{array}$ & $\begin{array}{c}18(8 \% / 12) \\
14(9 \% / 9) \\
5(5 \% / 3) \\
0(0 \%)\end{array}$ & $\begin{array}{c}30(4 \% / 21) \\
20(3 \% / 14) \\
7(5 \%) \\
1(7 \% / 0)\end{array}$ & $\begin{array}{l}48(1 \% / 17) \\
34(1 \% / 12) \\
12(2 \% / 4) \\
1(3 \% / 0)\end{array}$ & $\begin{array}{l}074 / 0 \mathrm{a} \\
263 / 0 \mathrm{a} \\
\mathrm{a} 547 / 0 \\
0.745 \mathrm{~b}\end{array}$ \\
\hline $\begin{array}{l}\text { White and red waste } \\
\text { 1. Danchestomatite } \\
\text { 2. Angular Chilitis } \\
\text { 3. Candida pseudomembranus } \\
\text { 4. Candida erythematosus } \\
\text { 5. Hairy tongue } \\
\text { 6. Language Atrophy } \\
\text { 7. Likinoid reaction } \\
\text { 8. Plane } \\
\text { 9. Leukoplakia } \\
\text { 10. keratose fescue } \\
\text { 11. Actinic Chylates } \\
\text { 12. Geographic languages }\end{array}$ & $\begin{array}{l}61(3 \% / / 43) \\
22(6 \% / 15) \\
15(6 \% / 10) \\
10(1 \% / 7) \\
6(3 \% / 4) \\
1(7 \% / 0) \\
6(3 \% / 4) \\
1(7 \% / 0) \\
0(0 \% / 0) \\
0(0 \% / 0) \\
0(0 \%) \\
0(0 \% / 0) \\
5(5 \% / 3)\end{array}$ & $\begin{array}{l}40(6 \% / / 28) \\
11(9 \% / 7) \\
4(9 \% / / 2) \\
11(9 \% / 7) \\
4(9 \% / / 2) \\
14(9 \% / 9) \\
9(4 \% / 6) \\
1(7 \% / 0) \\
1(7 \% / 0) \\
3(1 \% / 2) \\
3(1 \% / 2) \\
2(4 \% / 1) \\
9(4 \% / 6)\end{array}$ & $\begin{array}{l}101(9 \% / / 35) \\
33(7 \% / 11) \\
19(7 \% / 6) \\
21(5 \% / 7) \\
10(6 \% / 3) \\
15(3 \% / 5) \\
15(3 \% / 5) \\
2(7 \% / / 0) \\
1(3 \% / 0) \\
3(0 \% / 1) \\
3(0 \% / / 1) \\
2(7 \% / 0) \\
14(0 \% / 5)\end{array}$ & $\begin{array}{l}010 / 0^{\star} \mathrm{a} \\
044 / 0^{\star} \mathrm{a} \\
009 / 0^{\star} \mathrm{a} \\
807 / 0 \mathrm{a} \\
749 / 0 \mathrm{a} \\
001 / 0^{\star} \mathrm{a} \\
418 / 0 \mathrm{~b} \\
000 / 1 \mathrm{a} \\
498 / 0 \mathrm{~b} \\
122 / 0 \mathrm{~b} \\
247 / 0 \mathrm{~b} \\
247 / 0 \mathrm{~b} \\
267 / 0 \mathrm{a}\end{array}$ \\
\hline $\begin{array}{c}\text { Pigmented waste } \\
\text { 1. Physiological pigmentation } \\
\text { 2. Pulmonation caused by the drug } \\
\text { 3. Melanosis caused by cigarettes } \\
\text { 4. Tattoo amalgam } \\
\text { 5. Petya } \\
\text { 6. Oral melanocytic macula } \\
\text { 7.0ther }\end{array}$ & $\begin{array}{l}19(5 \% / / 13) \\
2(4 \% / 1) \\
1(7 \% / 0) \\
14(9 \% / 9) \\
0(0 \% / 0) \\
0(0 \% / 0) \\
1(7 \% / 0) \\
1(7 \% / 0)\end{array}$ & $\begin{array}{c}32(5 \% / / 22) \\
8(7 \% / 5) \\
0(0 \% / / 0) \\
6(3 \% / / 4) \\
15(7 \% / 10) \\
7(0 \% / / 5) \\
1(7 \% / 0) \\
1(7 \% / 0)\end{array}$ & $\begin{array}{l}51(0 \% / / 18) \\
10(6 \% / 3) \\
1(3 \% / / 0) \\
20(1 \% / 7) \\
15(3 \% / 5) \\
7(5 \% / / 2) \\
2(7 \% / 0) \\
2(7 \% / 0)\end{array}$ & $\begin{array}{c}041 / 0^{*} \mathrm{a} \\
060 / 0 \mathrm{a} \\
000 / 1 \mathrm{~b} \\
066 / 0 \mathrm{a} \\
000 / 0^{*} \mathrm{~b} \\
074 / 0 \mathrm{~b} \\
000 / 1 \mathrm{~b} \\
000 / 1\end{array}$ \\
\hline
\end{tabular}

$a$ : chi square test; b: Fisher exact test

Table 4 - The prevalence of oral lesions and denture information of the studied elderly

\begin{tabular}{|c|c|c|c|c|c|c|}
\hline Denture information & Resident & Non-resident & Total & $\mathrm{p}$-value & Lesions & p-value \\
\hline $\begin{array}{c}\text { Denture type } \\
\text { 1. Without tooth } \\
\text { 2. Complete denture } \\
\text { 3. With tooth/without partial denture } \\
\text { 4. With partial denture } \\
\text { 5. More than } 20 \text { natural teeth } \\
\text { 6. Other type of prosthesis }\end{array}$ & $\begin{array}{c}5(6 \% / / 3) \\
60(9 \% / / 42) \\
27(3 \% / 19) \\
14(0 \% / 10) \\
31(1 \% / 22) \\
3(1 \% / 2)\end{array}$ & $\begin{array}{c}24(1 \% / 17) \\
62(3 \% / 44) \\
36(7 \% / / 25) \\
6(3 \% / 4) \\
11(9 \% / / 7) \\
1(7 \% / 0)\end{array}$ & $\begin{array}{c}29(4 \% / 10) \\
122(6 \% / 43) \\
63(5 \% / 22) \\
20(1 \% / 7) \\
42(0 \% / 15) \\
4(4 \% / 1)\end{array}$ & $\mathrm{a} 000 / 0^{*}$ & $\begin{array}{c}14(3 \% / 48) \\
83(0 \% / 68) \\
22(9 \% / 34) \\
7(0 \% / 35) \\
24(0 \% / 57) \\
3(0 \% / 75)\end{array}$ & $\mathrm{a} 000 / 0^{*}$ \\
\hline $\begin{array}{c}\text { Duration of denture use } \\
\text { 1. Less than } 5 \text { years } \\
2.5-10 \text { years } \\
3.10-15 \text { years } \\
\text { 4. Over } 15 \text { years }\end{array}$ & $\begin{array}{c}15(4 \% / 22) \\
9(4 \% / 13) \\
18(9 \% / 26) \\
25(3 \% / 37)\end{array}$ & $\begin{array}{l}25(1 \% / 32) \\
14(9 \% / 17) \\
16(5 \% / 20) \\
23(5 \% / 29)\end{array}$ & $\begin{array}{l}40(6 \% / 27) \\
23(9 \% / 15) \\
34(4 \% / 23) \\
48(1 \% / 33)\end{array}$ & $126 / 0^{*} \mathrm{C}$ & $\begin{array}{l}26(0 \% / 65) \\
12(2 \% / / 52) \\
26(5 \% / 76) \\
28(3 \% / / 58)\end{array}$ & $228 / 0 c$ \\
\hline $\begin{array}{c}\text { Denture wash } \\
\text { 1. Failure to wash } \\
\text { 2. Wash with water } \\
\text { 3. Wash with toothbrush }\end{array}$ & $\begin{array}{l}18(3 \% / / 27) \\
26(4 \% / 39) \\
22(3 \% / 33)\end{array}$ & $\begin{array}{c}3(9 \% / 3) \\
33(9 \% / 42) \\
41(2 \% / 53)\end{array}$ & $\begin{array}{l}21(7 \% / / 14) \\
59(3 \% / / 41) \\
63(1 \% / 44)\end{array}$ & $000 / 0^{\star} \mathrm{a}$ & $\begin{array}{l}19(5 \% / / 90) \\
39(1 \% / 66) \\
36(1 \% / 57)\end{array}$ & $020 / 0^{\star} \mathrm{a}$ \\
\hline
\end{tabular}

a: chi square test; b: mann-whitney test 
Table 5 - The prevalence of oral lesions and hygienics of the studied elderly

\begin{tabular}{|c|c|c|c|c|c|c|}
\hline Denture information & Resident & Non-resident & Total & p-value & Lesions & p-value \\
\hline $\begin{array}{l}\text { Brushing times } \\
\text { 1. Irregular or never } \\
\text { 2. Once a week } \\
\text { 3.2-3 times a week } \\
\text { 4. Once a day } \\
\text { 5. More than once a day }\end{array}$ & $\begin{array}{c}92(1 \% / 68) \\
8(9 \% / 5) \\
5(7 \% / 3) \\
22(3 \% / 16) \\
8(9 \% / 5)\end{array}$ & $\begin{array}{c}75(3 \% / 54) \\
4(9 \% / 2) \\
14(1 \% / / 10) \\
35(4 \% / 25) \\
10(6 \% / 6)\end{array}$ & $\begin{array}{c}167(2 \% / 61) \\
12(6 \% / 43) \\
19(0 \% / 7) \\
57(9 \% / 20) \\
18(6 \% / 6)\end{array}$ & $\mathrm{CO} 020 / 0^{\star}$ & $\begin{array}{c}97(0 \% / 58) \\
3(0 \% / / 25) \\
6(5 \% / 31) \\
28(1 \% / 49) \\
12(6 \% / 66)\end{array}$ & c238/0 \\
\hline $\begin{array}{c}\text { Wash mouth in overnight } \\
\text { 1. Never } \\
\text { 2. Once } \\
\text { 3. Twice } \\
\text { 4. Three times } \\
\text { 5. More than three times }\end{array}$ & $\begin{array}{c}15(6 \% / 10) \\
31(0 \% / 22) \\
41(1 \% / 29) \\
40(4 \% / 28) \\
14(9 \% / 9)\end{array}$ & $\begin{array}{l}12(6 \% / 8) \\
28(0 \% / 20) \\
22(7 \% / 15) \\
51(4 \% / 36) \\
27(3 \% / 19)\end{array}$ & $\begin{array}{l}27(6 \% / 9) \\
59(0 \% / 21) \\
63(4 \% / 22) \\
91(4 \% / 32) \\
41(6 \% / 14)\end{array}$ & $000 / 0^{*} \mathrm{C}$ & $\begin{array}{l}18(6 \% / 66) \\
26(0 \% / 44) \\
39(9 \% / 61) \\
48(7 \% / 52) \\
22(6 \% / 53)\end{array}$ & $838 / 0 \mathrm{c}$ \\
\hline $\begin{array}{l}\text { Last visit to dentist } \\
\text { 1. In past year } \\
\text { 2. In past two years } \\
\text { 3. In last } 5 \text { years } \\
\text { 4. Over last5 years } \\
\text { 5. I do not know }\end{array}$ & $\begin{array}{c}33(7 \% / 23) \\
9(5 \% / 6) \\
16(5 \% / 11) \\
48(5 \% / 34) \\
32(0 \% / 23)\end{array}$ & $\begin{array}{c}47(6 \% / 33) \\
23(4 \% / 16) \\
26(6 \% / 18) \\
38(1 \% / 27) \\
6(3 \% / 4)\end{array}$ & $\begin{array}{c}80(7 \% / 28) \\
32(5 \% / / 1) \\
42(1 \% / 15) \\
86(8 \% / 30) \\
38(6 \% / 13)\end{array}$ & $000 / 0^{\star} \mathrm{C}$ & $\begin{array}{l}41(2 \% / / 51) \\
12(5 \% / 37) \\
24(1 \% / / 57) \\
58(4 \% / 67) \\
17(7 \% / 44)\end{array}$ & $250 / 0 c$ \\
\hline $\begin{array}{l}\text { Cause of last visit to dentist } \\
\text { 1. The pain } \\
\text { 2. Normal treatments } \\
\text { 3. Periodic examination } \\
\text { 4.I do not know }\end{array}$ & $\begin{array}{c}36(9 \% / 25) \\
54(8 \% / 38) \\
11(9 \% / 7) \\
38(3 \% / 27)\end{array}$ & $\begin{array}{c}22(8 \% / 115) \\
101(7 \% / 72) \\
2(4 \% / 1) \\
14(1 \% / 10)\end{array}$ & $\begin{array}{c}58(9 \% / 20) \\
155(8 \% / / 55) \\
13(7 \% / 4) \\
52(7 \% / 18)\end{array}$ & $000 / 0^{* a}$ & $\begin{array}{c}32(1 \% / 55) \\
90(0 \% / / 58) \\
5(4 \% / 38) \\
25(0 \% / 48)\end{array}$ & $389 / 0 a$ \\
\hline
\end{tabular}

a: chi square test; b: mann-whitney test

\section{DISCUSSION}

Health and oral lesions information of elderly can be a good guide to creating comprehensive plans for solving problems and improving oral health and quality of life in elderly. Therefore, many studies have been conducted to determine the prevalence of oral lesions in the elderly $[9,10]$.

The results of this study showed that $55.3 \%$ resident nursing home and 53.6\% nonresident were diagnosed with at least one oral lesion. The prevalence of lesions in the resident nursing home was higher than non-residents, but their difference was not significant. In a study by Saintrain et a., the prevalence of oral lesions in elderly was reported $47 \%$, which in resident nursing home was more than nonresident, but no significant difference was observed [11]. These results may reflect our misconception about low level of oral lesions of non-resident. The high prevalence of oral lesions in elderly can be due to involvement of elderly in daily work, and ignorance and neglect to oral health. In several studies by Molania et al. [5], Maleki et al. [10] and Motalebnezhad et al. [12] in Iran, the prevalence of oral lesion was reported $100 \%, 77 \%$ and $84 \%$, respectively. The lower incidence of lesions in present study than other can be due to difference in place of sampling; so that sampling was performed in some studies in dental clinics. Also, in some studies sublingual varices and fissured tongue have been considered as oral lesions, while these cases are oral anatomical norms and have not been evaluated in our study. The cultural differences, oral habits, educational level, and even genetic differences can be the cause of these differences [13].

The most common lesions of oral mucosa in the present study were related to epulis ficuratum (12.1\%), dancharostomyitis $(11.9 \%)$ and candidiasis (11.2\%). The more prevalence of lesions, such as epulis ficuratum, denture stomatitis and candidiasis are due to inappropriate dentures, unsatisfactory health and irregular oral examination [11, 14]. These results are consistent with studies conducted 
in many countries such as Brazil, Germany, Taiwan. The higher and significant incidence of denture stomatitis in residents nursing home $(15.6 \%)$ than non-resident $(7.9 \%)$ can indicate unsatisfactory health in the resident nursing home. In contrast, with regard to higher incidence of epulis fissuratum in the non-resident (14.3\%) compared to the elderly (9.9\%), it can be concluded that inappropriate dentures are also high in the non-resident. In general, according to obtained results, due to low level of health in the resident nursing home, denture stomatitis has a high prevalence. On the other hand, the presence of inappropriate dentures in the non-resident causes an increase in the epididymis fissuretoma.

The most frequent oral lesions in residents and non-residents was related to white and red lesions (35.9\%). Also, in Babae et al. study, the most common lesions were related to white and red lesions (36.3\%) [15]. Also, in present study the prevalence of white and red lesions was significantly higher in residents nursing home. In contrast, the prevalence of pigmented lesions was significantly higher in non-resident, which may be due to higher incidence of tattooed amalgam.

In the present study, there was a significant correlation between age and prevalence of lesions, which was consistent with Ferreira et al. and Babae et al. studies $[15,16]$. This relationship seems logical, because the prevalence of malignant and pre-malignant lesions increases with age increasing. Also, many oral lesions are associated with drug use, various prostheses and systemic diseases in elderly [17].

In this study, there was no relationship between gender and frequency of oral lesions. In some studies, a higher incidence of lesions was reported in men $[5,18]$; but in the Mosqueda study, more lesions were showed in women [17]. However, in present study, there was a higher incidence of specific lesions in men and women. Therefore, oral lesions such as angular cheilitis, ulcers, herpes, peptic, tattoo amalgams were more in women, and lesions such as denture stomatitis, apolipse, candida, hairy tongue and melanosis were more men.

High consumption of cigarettes by men can be due to the prevalence of hairy tongue and melanosis caused by cigarettes. Also, prevalence of hair loss, candida, denture stomatitis and epilepsy fissuratum in men can be due to poor health in men and lack of attention to oral health. Whereas the higher prevalence of tattooed amalgam in women may be due to women's greater importance to oral health.

In the present study, $54.0 \%$ of the elderly were without teeth $(61.4 \%$ in resident nursing home vs $46.5 \%$ in non-resident), which $80.7 \%$ had complete dentures. The levels of toothlessness in this study was more than that observed in Sri Lanka (17\%) and Zagreb $(45.3 \%) \quad[19,20]$. Although health education policies and dental care promotion programs have been carried out in elderly in recent years, but tooth lessens in elderly population also is very common. According to the WHO statement, half of elderly with 65-75-year-old, should have more than 20 natural teeth [11], while in our study it is $6.8 \%$.

Blood pressure $(38.3 \%)$ was the most common systemic disorder in both elderly groups. Also, cardiovascular disease (23.2\%) and diabetes $(18.9 \%)$ had a high prevalence in the studied elderly. The most commonly used drug in the elderly was related to antihypertensive drugs. These types of drugs can have side effects such as oral ulcers, swelling or salivary gland pain, gum hyperplasia and angioedema. The psychological disorders such as dementia were the second most commonly disease in the residents nursing home. Psychological interventions can affect on oral hygiene behaviors. Therefore, the higher prevalence of psychological disease in residents nursing home can be explained the lower health in them.

\section{Strength and limitations of this study}

One of the limitations in the present study was high age of the participants, which made it difficult to communicate and receive accurate answers. Also, the impossibility of biopsy of 
suspected lesions caused uncertainty about separation and classification of some lesions, especially white plates.

\section{CONCLUSION}

In general, our study showed that prevalence of oral mucosal lesions in resident nursing home is slightly higher than nonresident. The presence of agents such as inappropriate dentures and poor oral hygiene in resident nursing home caused a lot of oral lesions, such as denture stomatitis. Therefore, proper maintenance and sanitation of dentures is essential for prevention and control of lesions, especially in resident nursing home. In addition, regular examination can play a prominent role in early diagnosis and better prevention of oral lesions In elderly.

\section{REFERENCES}

1. AmadoriF,Bardellini E,ContiG, Majorana A. Oral mucosal lesions in teenagers: a cross-sectional study. Ital J Pediatr. 2017 May 31;43(1):50. doi: 101186/s13052017-0367-7.

2. Tortorici S, Corrao S, Natoli G, Difalco P.Prevalence and distribution of oral mucosal non-malignant lesions in the western Sicilian population. Minerva Stomatol. 2016 Aug;65(4):191-206.

3. Mirzaei M, Shams-Ghahfarkhi M. Demographic characteristics of the elderly population in Iran according to the census 1976-2006. Iran J Ageing. 2007:2(3):326-31.

4. Nasirpour H, Key YA, Kazemipur N, Shadman B, Hajazimian S, Taefehshokr $\mathrm{S}$. The effects of cholesterol lowering drugs on vitamin $\mathrm{D}$ status in familial hypercholesterolemia patients. Arch Med Lab Sci. 2017;3(4):29-33.

5. Molania T,Mousavi SJ, Ahangari M, Salehi M. Prevalence of Oral Mucosal Lesions in Nursing Home Residents. J Mazandaran Univ Med Sciences. 2016;26(137):73-81

6. Strayer M. Oral health care for homebound and institutional elderly.J Calif Dent Assoc. 1999 Sep;27(9):703-8.
7. Pfrimer K, Marques Messias M, Ferriolli E, Vieira Salles MS, Roma Junior LC, Saran Netto A, etal. Assessment, evaluation and nutrition monitoring in older people living in a rest home. Arch Latinoam Nutr.2015 Jun;65(2):104-9.

8. Van Lancker A, Verhaeghe S, Van Hecke A, Vanderwee K, Goossens J, Beeckman D. The association between malnutrition and oral health status in elderly inlong-term care facilities: a systematic review. Int J Nurs Stud. 2012 Dec;49(12):1568-81. doi: 10.1016/j.jinurstu.2012.04.001.

9. Coleman P.Improving oral health care for the frail elderly: a review of widespread problems and best practices. Geriatr Nurs. 2002 JulAug;23(4):189-99.

10. MalekiZ,Ghaem maghami A, Lesan S. Comparison of soft tissue lesions (OSTLS) prevalence in elderly institutionalized population who are resided in private and governmental institutes in Tehran Iran 2001-2003. Shahid Beheshti Univ Dent J.2006;23(4):663-9.

11. Saintrain MV, Almeida CB, Naruse TM, Gonçalves VP.Oral lesions in elderly patients of a community in Brazilian Northeast. Gerodontology.2013 Dec;30(4):283-7.doi: 10.1111/.1741-2358.2012.00680.x. Epub 2012 May 27.

12. Motalebnezhad M, Shirvani M. Oral mucosal lesions in elderly population Tehran Kahrizak Geriatric Institute, 2000. J Babol Univ Med Sci. 2002.4(3):2833.

13. Isazadeh A, Azimian SH, Tariverdi N, Rahmani SA, Esmaeili M, Karimkhaniloue S, Mohammadoo-Khorasani M. Effects of coagulation factor XIII (Val34Leu) polymorphism on recurrent pregnancy loss in Iranian Azeri women. J Lab Med. 2017 Apr 10;41(2):89-92. doi: 10.1515/labmed-2017-0012

14. ReichartPA. Oral mucosal lesions in a representative cross-sectional study of aging Germans. Community Dent Oral Epidemiol. 2000 0ct,28(5):390-8.

15. Babae N, Khoshsirat A, Molania T. Frequency of Oral Mucosal Lesion in Patients Attending Babol Dental School, 2010. J Mazandaran Unive Med Sci. 2013.23(103):114-8.

16. Ferreira RC, Magalhaes CS, Moreira AN. oral mucosal alterations among the institutionalized elderly in brazil. Braz Oral Res. 2010 Jul-Sep;24(3):296-302

17. Mosqueda A, Diaz M, Velasquez M, Irigoyen M, Caballero S, SidaE. Prevalence of alterations of the oral mucosa in the elderly. I study in two groups of the south of the city of Mexico. Selected Topics Clin Res. 1998;4:39-50

18. Lin HC, CorbetEF, Lo EC. Oral mucosal lesions in adult Chinese.J DentRes. 2001May;80(5):1486-90.

19. Pallegedara C,Ekanayake L Tooth loss, the wearing of dentures and associated factors in SriLankan older individuals. Gerodontology. 2005 Dec;22(4):193-9.

20. Trulsson U, Engstrand P,Berggren U, NannmarkU, Branemark PI. Edentulousness and oral rehabilitation: experiences from the patients' perspective. Eur J Oral Sci. 2002 Dec;110(6):417-24.

\section{Fahimeh Pakravan}

(Corresponding address)

Dental Implant Research Center, Department of Oral Medicine, Dental

research institiute, Isfahan University of Medical Sciences, Isfahan, Iran.

Date submitted: 2019 Mar 07

E-mail: pakravan@dnt.mui.ac.ir 